\title{
Correlation Studies in Turmeric (Curcuma longa $\mathbf{L}$.)
}

\author{
H. Dev* and Vipin Sharma \\ Department of Vegetable Science, Dr YS Parmar University of Horticulture and Forestry, \\ Nauni, Solan 173 230, Himachal Pradesh, India \\ *Corresponding author
}

\section{A B S T R A C T}

\begin{tabular}{|l|}
\hline K e y w o r d s \\
$\begin{array}{l}\text { Turmeric, } \text { Curcuma } \\
\text { longa, Correlation } \\
\text { coefficient, Path } \\
\text { analysis, Quality, } \\
\text { Yield }\end{array}$ \\
\hline Article Info \\
\hline $\begin{array}{l}\text { Accepted: } \\
\text { 24 July 2020 } \\
\text { Available Online: } \\
\text { 10 August } 2020\end{array}$ \\
\hline
\end{tabular}

Forty turmeric genotypes collected from different parts of the country including two recommended varieties as checks viz. Palam Lalima and Palam Pitambar were planted at the Experimental Farm of the Department of Vegetable Science, Dr. YS Parmar University of Horticulture and Forestry, Nauni, Solan (HP) during Kharif season of 2011 and evaluated for different yield and yield contributing traits. The observations were recorded on emergence, number of tillers and leaves per plant, leaf length and breadth, plant girth and height, length, girth, core diameter and weight of mother, primary and secondary rhizomes, number of primary and secondary rhizomes per plant, yield per plant, per plot and per hectare, incidence of rhizome rot, dry matter and curcumin content. The correlation studies indicated that yield per plant was positively and significantly correlated with emergence, number of tillers and leaves per plant, leaf length, plant girth and height, length, core diameter and weight of mother, primary and secondary rhizomes, while significant negative correlations were observed with incidence of rhizome rot and curcumin content. Among all the traits studied, weight of primary rhizome had maximum positive direct effect on yield followed by length of primary rhizome, core diameter of mother rhizome, length and weight of secondary rhizome, emergence, plant girth and height.

\section{Introduction}

Turmeric (Curcuma longa L.) is one of the important spice crops in India belonging to the family Zingiberaceae and plays a vital role in the national economy. Probably, it has been originated in the slope of hills in the tropical forests of West Coast of South India (Stahl, 1980). Turmeric is very much identified with human civilization, religion, customs and it finds use both in developed and under developed countries. It is grown for underground stem called as rhizome, which is used to impart flavour and colour to foodstuffs after cleaning, drying, polishing and powdering. It is a principal ingredient in curry powder. Turmeric oleoresin is used in brine pickles and to some extent in nonalcoholic beverages, gelatins, butter and cheese, etc. The colour curcumin extracted from turmeric is used as a colorant. Turmeric is also used as a dye in textile industry. It is used in the preparation of medicinal oils, ointments and poultice. It is stomachic, 
carminative, tonic, blood purifier and an antiseptic. It is also used in cosmetics. The aqueous extracts have biopesticidal properties. India is largest producer, consumer and exporter of turmeric in the world and accounts for more than 50 per cent of the world trade (Chaudhary et al., 2006). In India, it is grown in area of 1, 93, 000 hectares with annual production of 10, 52, 000 tonnes (Anonymous, 2017). Out of the total turmeric produced in India 90 per cent is consumed locally. Remaining 10 per cent of the production is exported to various countries like US, UK, Middle East, Japan, Singapore, Malaysia, South Africa, Australia and other countries. Andhra Pradesh followed by Odisha, Tamil Nadu and Maharashtra states in India constitutes the lion's share in India's turmeric production (Maurya, 1990). In Himachal Pradesh, turmeric has not attained significant status among spice crops probably due to poor yield and being a long duration crop. However, some growers especially in lower hills have started showing interest in the crop and the area is steadily increasing as the crop can be successfully planted under rainfed conditions under minimal care and attention. Moreover, increasing monkey menace and engagement of farmers in other occupations offer better opportunities for increasing acreage under this crop in the state. Turmeric is a vegetatively propagated crop and the hybridization is practiced rarely in the areas where flowering occurs. Because of this nature of the crop it devoid the conventional breeding approaches like hybridization, therefore, selection is the easiest method of improving the crop other than mutation and polyploidy breeding. Association of plant characters has always been helpful as a basis for selecting desired genotypes. The traits that contribute to yield can be identified by correlation and path coefficient analysis. Correlation analysis followed by the path coefficient analysis provides a true picture of genetic association among different traits.
Correlation in combination with the path coefficient analysis quantifies the direct and indirect contribution of one character upon another. Considering these aspects and importance of turmeric, the present study was conducted to estimate the correlation of yield with other characters, and direct and indirect effect of important characters on yield through path coefficient analysis in order to develop selection criteria for improving rhizome yield potentiality of turmeric.

\section{Materials and Methods}

The present investigations were carried out at the Experimental Farm of the Department of Vegetable Science, Dr. YS Parmar University of Horticulture and Forestry, Nauni, Solan (HP) during Kharif season of 2011. Forty diverse genotypes of turmeric collected from different parts of the country including two recommended varieties as checks viz. Palam Lalima and Palam Pitambar were used for the present investigations. Uniform size of rhizomes were directly sown in the field in the month of April, 2011 at a spacing of $30 \mathrm{x}$ $20 \mathrm{~cm}$ in raised beds of $3 \times 1 \mathrm{~m}$ size, accommodating 50 plants per plot. Drainage channels were also made between plots. Each collection was sown in a Randomized Block Design with three replications. The standard cultural practices recommended in the Package of Practices for Vegetable Crops, were followed to ensure a healthy crop stand (Anonymous, 2009). Data were recorded from the mean of ten plants tagged randomly from each genotype in each replication on different characters, viz. emergence, number of tillers and leaves per plant, leaf length, plant girth and height, length, core diameter and weight of mother, primary and secondary rhizomes, incidence of rhizome rot, dry matter recovery, curcumin content and yield per plant. The genotypic and phenotypic correlations were calculated as per Al-Jibouri et al., (1958) by using analysis of variance and covariance 
matrix in which total variability has splited into replications, genotypes and errors. The genotypic and phenotypic correlation coefficients were used in finding out their direct and indirect contribution towards yield per plant. The direct and indirect paths were obtained by following Dewey and Lu (1959).

\section{Results and Discussion}

\section{Correlation studies}

Yield is a complex character and associated with several yield contributing characters. Selection for yield as such may not be effective unless other yield components influencing it directly or indirectly are taken into consideration. Knowledge of degree of association of yield with its components is of great importance, because yield is not an independent character, but it is the resultant of the interactions of a number of component characters among themselves as well as with the environment in which the plant grow. Further each character is likely to be modified by action of genes present in the genotypes of plant and also by the environment and it becomes difficult to evaluate this complex character directly.

When selection pressure is exercised for improvement of any character highly associated with yield, it simultaneously affects a number of other correlated traits. Hence, the knowledge regarding association of characters with yield and among themselves provides a guideline to the plant breeder for making improvement through selection. Therefore, correlation study of yield with its component traits has been executed, to find out the yield contributing traits. In most of the traits, the genotypic correlation coefficients were slightly higher in magnitude than the phenotypic correlation coefficients indicating a fairly strong association between the characters studied (Table 1).
The correlation coefficients among the different characters were worked out at phenotypic and genotypic levels. In general, the genotypic correlation coefficients were higher in magnitude than phenotypic correlation coefficients, indicating a strong inherent association among the traits.

The phenotypic and genotypic correlation coefficients among different characters showed that yield per plant had significantly positive association with emergence, number of tillers and leaves per plant, leaf length, plant girth and height, length, core diameter and weight of mother, primary and secondary rhizomes, while significantly negative correlations were observed with incidence of rhizome rot and curcumin content. Similar correlations of yield with various other horticultural traits to different extents in turmeric have been reported by Pathania et al., (1981), Jalgaonkar et al., (1990), Nandi et al., (1994), Shashidhar and Sulikeri (1997), Shashidhar et al., (1997), Lynrah et al., (1998), Venkatesha et al., (1998), Chandra et al., (1999), Jana et al., (2001), Raveendra et al., (2001), Panja et al., (2002), Prasad et al., (2004), Rao et al., (2004), Tomar et al., (2005), Kumar et al., (2006), Yadav et al., (2006), Kumar et al., (2007), Velmurugan et al., (2008), Sharon et al., (2011) and Singh et al., (2012).

\section{Path coefficient analysis}

Although correlation studies are helpful in determining the components of yield but it does not provide a clear picture of nature and extent of contributions made by number of independent traits. Path coefficient analysis devised by Dewey and Lu (1959), however, provides a realistic basis for allocation of appropriate weightage to various attributes while designing a pragmatic programme for the improvement of yield. 
Table.1 Phenotypic and genotypic coefficients of correlation among different traits in turmeric

\begin{tabular}{|c|c|c|c|c|c|c|c|c|c|c|c|c|c|c|c|c|c|c|c|c|}
\hline Traits & & EMG & NTP & NLP & $\mathbf{L L}$ & PG & PH & LMR & CMR & WMR & LPR & CPR & WPR & LSR & CSR & WSR & IRR & DMR & $\mathrm{CC}$ & YPP \\
\hline \multirow[t]{2}{*}{ EMG } & $\mathbf{P}$ & 1.000 & $0.997 * *$ & -0.040 & 0.126 & $0.997 * *$ & -0.024 & $0.250^{* *}$ & $0.244^{* *}$ & $0.242^{* *}$ & $0.303^{* *}$ & $0.298 * *$ & $0.310^{* *}$ & 0.165 & 0.176 & 0.148 & $-0.954 * *$ & $-0.186^{*}$ & 0.022 & $0.316^{* *}$ \\
\hline & G & 1.000 & $0.999 * *$ & -0.029 & 0.074 & $0.999 * *$ & -0.045 & $0.252^{* * *}$ & $0.257^{* *}$ & $0.279 * *$ & 0.431 ** & $0.435^{* *}$ & $0.441^{* *}$ & $0.214^{*}$ & $0.212^{*}$ & 0.155 & $-0.999 * *$ & $-0.224 *$ & 0.025 & $0.437 * *$ \\
\hline \multirow[t]{2}{*}{ NTP } & $\mathbf{P}$ & & 1.000 & -0.042 & 0.125 & 0.995 ** & -0.025 & $0.247 * *$ & $0.240^{* * *}$ & 0.239 ** & $0.301 * *$ & $0.294 * *$ & $0.308^{* *}$ & 0.164 & 0.178 & 0.148 & $-0.953 * *$ & $-0.186^{*}$ & 0.021 & $0.314 * *$ \\
\hline & G & & 1.000 & -0.027 & 0.076 & $0.998^{* *}$ & -0.043 & $0.254^{* *}$ & $0.261^{* *}$ & $0.281^{* *}$ & $0.433^{* *}$ & $0.438^{* *}$ & $0.443^{* *}$ & $0.215^{*}$ & $0.209^{*}$ & 0.155 & $-0.999 * *$ & $-0.224^{*}$ & 0.026 & $0.439 * *$ \\
\hline \multirow[t]{2}{*}{ NLP } & $\mathbf{P}$ & & & 1.000 & $0.816^{* * *}$ & -0.034 & $0.985^{* *}$ & $0.392 * *$ & $0.395^{* *}$ & $0.297 * *$ & $0.228^{*}$ & $0.230^{*}$ & 0.167 & 0.098 & 0.094 & 0.076 & 0.024 & $0.328 * *$ & $-0.395 * *$ & 0.169 \\
\hline & G & & & 1.000 & 0.997 ** & -0.031 & $0.995^{* *}$ & $0.495^{* *}$ & $0.494 * *$ & $0.360^{* *}$ & $0.264 * *$ & $0.263^{* *}$ & 0.183 & 0.165 & 0.170 & 0.138 & 0.095 & $0.547 * *$ & $-0.636^{* *}$ & $0.198^{*}$ \\
\hline \multirow[t]{2}{*}{$\mathbf{L L}$} & $\mathbf{P}$ & & & & 1.000 & 0.130 & $0.841^{* *}$ & $0.401 * *$ & $0.402 * *$ & $0.315^{* *}$ & $0.335 * *$ & $0.336^{* *}$ & $0.276^{* *}$ & 0.150 & 0.147 & 0.134 & -0.129 & $0.348^{* *}$ & $-0.378 * *$ & $0.281 * *$ \\
\hline & G & & & & 1.000 & 0.076 & $0.994 * *$ & $0.526 * *$ & $0.525 * *$ & $0.412^{* *}$ & $0.383 * *$ & $0.383^{* *}$ & $0.306^{* *}$ & $0.222 *$ & $0.222 *$ & $0.211^{*}$ & -0.020 & $0.542 * *$ & $-0.599 * *$ & $0.327 * *$ \\
\hline \multirow[t]{2}{*}{ PG } & $\mathbf{P}$ & & & & & 1.000 & -0.019 & $0.252 * *$ & $0.248^{* *}$ & $0.244 * *$ & $0.305 * *$ & $0.301 * *$ & $0.312^{* *}$ & 0.166 & 0.174 & 0.148 & $-0.953 * *$ & -0.182 & 0.015 & $0.317 * *$ \\
\hline & G & & & & & 1.000 & -0.046 & $0.245^{* * *}$ & $0.249^{* *}$ & $0.271^{* *}$ & $0.422 * *$ & $0.425^{* * *}$ & $0.431^{* *}$ & $0.209^{*}$ & $0.208^{*}$ & 0.153 & $-0.991 * *$ & $-0.212 *$ & 0.021 & $0.428 * *$ \\
\hline \multirow[t]{2}{*}{ PH } & $\mathbf{P}$ & & & & & & 1.000 & $0.392 * *$ & $0.395^{* *}$ & $0.293 * *$ & $0.234^{*}$ & $0.237^{*}$ & 0.173 & 0.108 & 0.102 & 0.083 & 0.018 & $0.340 * *$ & $-0.409 * *$ & 0.176 \\
\hline & G & & & & & & 1.000 & $0.492 * *$ & $0.491 * *$ & $0.360^{* *}$ & $0.258^{* *}$ & $0.257^{* *}$ & 0.177 & 0.157 & 0.163 & 0.132 & 0.099 & $0.534 * *$ & $-0.621 * *$ & $0.192 *$ \\
\hline \multirow[t]{2}{*}{ LMR } & $\mathbf{P}$ & & & & & & & 1.000 & $0.988^{* *}$ & $0.950 * *$ & $0.660 * *$ & 0.660 ** & $0.631^{* *}$ & $0.574 * *$ & $0.573 * *$ & $0.561^{* *}$ & $-0.269 * *$ & -0.062 & $-0.278 * *$ & $0.705^{* *}$ \\
\hline & G & & & & & & & 1.000 & $0.994 * *$ & $0.959^{* *}$ & 0.771 ** & $0.772 * *$ & $0.742 * *$ & $0.723 * *$ & $0.725 * *$ & $0.703^{* *}$ & $-0.256 * *$ & -0.053 & $-0.336 * *$ & $0.838 * *$ \\
\hline \multirow[t]{2}{*}{ CMR } & $\mathbf{P}$ & & & & & & & & 1.000 & 0.950 ** & $0.658 * *$ & $0.663^{* *}$ & $0.630^{* *}$ & $0.573 * *$ & 0.566 ** & $0.557^{* *}$ & $-0.265^{* *}$ & -0.061 & $-0.275^{* *}$ & $0.704 * *$ \\
\hline & G & & & & & & & & 1.000 & 0.960 ** & $0.733 * *$ & $0.771^{* *}$ & $0.742 * *$ & $0.724 * *$ & $0.730^{* *}$ & $0.706^{* *}$ & $-0.260 * *$ & -0.054 & $-0.337 * *$ & $0.839 * *$ \\
\hline \multirow[t]{2}{*}{ WMR } & $\mathbf{P}$ & & & & & & & & & 1.000 & $0.648 * *$ & 0.650 ** & $0.640 * *$ & $0.488^{* *}$ & $0.483 * *$ & $0.489 * *$ & $-0.251^{* *}$ & -0.073 & $-0.215 *$ & $0.696 * *$ \\
\hline & G & & & & & & & & & 1.000 & 0.760 ** & 0.759 ** & 0.760 ** & $0.604 * *$ & $0.606^{* *}$ & $0.604 * *$ & $-0.275^{* *}$ & -0.080 & $-0.265^{* *}$ & $0.832 * *$ \\
\hline \multirow[t]{2}{*}{ LPR } & $\mathbf{P}$ & & & & & & & & & & 1.000 & $0.998^{* *}$ & $0.990^{* *}$ & $0.362^{* *}$ & $0.354 * *$ & $0.335^{* * *}$ & $-0.322 * *$ & 0.152 & $-0.241 * *$ & $0.975^{* *}$ \\
\hline & G & & & & & & & & & & 1.000 & $0.999 * *$ & $0.995^{* *}$ & $0.368^{* *}$ & $0.363^{* *}$ & $0.335^{* *}$ & $-0.423 * *$ & $0.190^{*}$ & $-0.276^{* *}$ & $0.983 * *$ \\
\hline \multirow[t]{2}{*}{ CPR } & $\mathbf{P}$ & & & & & & & & & & & 1.000 & $0.989 * *$ & $0.362 * *$ & $0.348 * *$ & $0.333^{* *}$ & $-0.317 * *$ & 0.153 & $-0.238^{*}$ & $0.974 * *$ \\
\hline & G & & & & & & & & & & & 1.000 & $0.995^{* *}$ & $0.369^{* *}$ & $0.367^{* * *}$ & $0.337^{* *}$ & $-0.426^{* *}$ & $0.190^{*}$ & $-0.277 * *$ & $0.983^{* *}$ \\
\hline
\end{tabular}




\begin{tabular}{|c|c|c|c|c|c|c|c|c|c|c|c|c|c|c|c|c|c|c|c|c|}
\hline Traits & & EMG & NTP & NLP & $\mathbf{L L}$ & PG & PH & LMR & CMR & WMR & LPR & CPR & WPR & LSR & CSR & WSR & IRR & DMR & $\mathrm{CC}$ & YPP \\
\hline \multirow[t]{2}{*}{ WPR } & $\mathbf{P}$ & & & & & & & & & & & & 1.000 & $0.315^{* *}$ & $0.305^{* *}$ & $0.290^{* *}$ & $\begin{array}{c}- \\
0.328^{* *}\end{array}$ & 0.127 & -0.179 & $0.973 * *$ \\
\hline & G & & & & & & & & & & & & 1.000 & $0.313^{* *}$ & $0.308^{* *}$ & $0.282 * *$ & $\stackrel{-}{0.434^{* *}}$ & 0.162 & $-0.212^{*}$ & $0.975^{* *}$ \\
\hline \multirow[t]{2}{*}{ LSR } & $\mathbf{P}$ & & & & & & & & & & & & & 1.000 & $0.992 * *$ & $0.985^{* *}$ & -0.164 & $-0.251 * *$ & -0.166 & $0.523 * *$ \\
\hline & G & & & & & & & & & & & & & 1.000 & $0.997 * *$ & $0.988^{* *}$ & $\stackrel{-}{-}$ & $-0.311 * *$ & $-0.194 *$ & $0.513^{* *}$ \\
\hline CSR & G & & & & & & & & & & & & & & 1.000 & $0.987 * *$ & $\stackrel{-}{0.241 * *}$ & $-0.321 * *$ & -0.181 & $0.509 * *$ \\
\hline \multirow[t]{2}{*}{ WSR } & $\mathbf{P}$ & & & & & & & & & & & & & & & 1.000 & -0.145 & $-0.235^{*}$ & -0.180 & $0.504 * *$ \\
\hline & G & & & & & & & & & & & & & & & 1.000 & -0.181 & $-0.289 * *$ & $-0.221^{*}$ & $0.488^{* *}$ \\
\hline \multirow[t]{2}{*}{ IRR } & $\mathbf{P}$ & & & & & & & & & & & & & & & & 1.000 & $0.260 * *$ & -0.049 & $-0.331 * *$ \\
\hline & G & & & & & & & & & & & & & & & & 1.000 & $0.290^{* *}$ & -0.077 & $-0.437 * *$ \\
\hline DMR & $\mathbf{P}$ & & & & & & & & & & & & & & & & & 1.000 & $-0.355^{* *}$ & 0.058 \\
\hline \multirow[t]{2}{*}{$\mathrm{CC}$} & $\mathbf{P}$ & & & & & & & & & & & & & & & & & & 1.000 & $-0.205^{*}$ \\
\hline & G & & & & & & & & & & & & & & & & & & 1.000 & $-0.244 * *$ \\
\hline \multirow[t]{2}{*}{ YPP } & $\mathbf{P}$ & & & & & & & & & & & & & & & & & & & 1.000 \\
\hline & G & & & & & & & & & & & & & & & & & & & 1.000 \\
\hline
\end{tabular}

*Significant at $5 \%$ level of significance

$* *$ Significant at $1 \%$ level of significance

Where, $\mathrm{EMG}=$ Emergence, $\mathrm{NTP}=$ Number of tillers per plant, $\mathrm{NLP}=$ Number of leaves per plant, $\mathrm{LL}=$ Leaf length, $\mathrm{PG}=\mathrm{Plant}$ girth, $\mathrm{PH}=\mathrm{Plant}$ height, $\mathrm{LMR}=\mathrm{Length}$ of mother rhizome, $\mathrm{CMR}=$

Core diameter of mother rhizome, WMR= Weight of mother rhizome, $\mathrm{LPR}=$ Length of primary rhizome, $\mathrm{CPR}=\mathrm{Core}$ diameter of primary rhizome, WPR $=$ Weight of primary rhizome, $\mathrm{LSR}=$

Length of secondary rhizome, CSR= Core diameter of secondary rhizome, WSR= Weight of secondary rhizome, IRR= Incidence of rhizome rot, DMR= Dry matter recovery, CC= Curcumin content and YPP= Yield per plant 
Table.2 Estimates of direct and indirect effects of different traits on yield of turmeric

\begin{tabular}{|c|c|c|c|c|c|c|c|c|c|c|c|c|c|c|c|c|c|c|c|c|}
\hline Traits & & EMG & NTP & NLP & $\mathbf{L L}$ & PG & PH & LMR & CMR & WMR & LPR & CPR & WPR & LSR & CSR & WSR & IRR & DMR & $\mathrm{CC}$ & CYPP \\
\hline \multirow{2}{*}{ EMG } & $\mathbf{P}$ & 0.142 & -0.138 & 0.000 & 0.000 & -0.004 & 0.000 & 0.015 & -0.015 & 0.000 & 0.009 & -0.009 & 0.280 & 0.001 & -0.001 & 0.036 & 0.000 & 0.000 & 0.000 & $0.316 * *$ \\
\hline & G & 0.072 & -0.102 & 0.000 & 0.000 & 0.036 & 0.000 & -0.061 & 0.065 & -0.002 & 0.190 & -0.190 & 0.400 & 0.010 & -0.010 & 0.036 & -0.005 & 0.000 & 0.000 & $0.437 * *$ \\
\hline \multirow[t]{2}{*}{ NTP } & $\mathbf{P}$ & 0.141 & -0.138 & 0.000 & 0.000 & -0.004 & 0.000 & 0.015 & -0.014 & 0.000 & 0.009 & -0.009 & 0.278 & 0.001 & -0.001 & 0.036 & 0.000 & 0.000 & .000 & $0.314 * *$ \\
\hline & G & 0.072 & -0.101 & 0.000 & 0.000 & 0.036 & 0.000 & -0.061 & 0.065 & -0.002 & 0.190 & -0.193 & 0.402 & 0.010 & -0.010 & 0.036 & -0.005 & 0.000 & 0.000 & $0.439 * *$ \\
\hline \multirow[t]{2}{*}{ NLP } & $\mathbf{P}$ & -0.006 & 0.006 & 0.001 & 0.000 & 0.000 & -0.001 & 0.024 & -0.024 & 0.000 & 0.006 & -0.007 & 0.151 & 0.000 & 0.000 & 0.018 & 0.000 & 0.000 & 0.000 & 0.169 \\
\hline & G & -0.002 & 0.003 & -0.003 & -0.001 & -0.001 & 0.002 & -0.119 & 0.124 & -0.002 & 0.116 & -0.116 & 0.166 & 0.008 & -0.008 & 0.032 & 0.001 & 0.000 & -0.001 & $0.198^{*}$ \\
\hline \multirow[t]{2}{*}{ LL } & $\mathbf{P}$ & 0.018 & -0.017 & 0.000 & 0.000 & 0.000 & 0.000 & 0.024 & -0.024 & 0.000 & 0.010 & -0.010 & 0.249 & 0.001 & -0.001 & 0.032 & 0.000 & 0.000 & 0.000 & $0.281^{* *}$ \\
\hline & G & 0.005 & -0.008 & -0.003 & -0.001 & 0.003 & 0.002 & -0.126 & 0.132 & -0.003 & 0.169 & -0.168 & 0.278 & 0.010 & -0.011 & 0.049 & 0.000 & 0.000 & -0.001 & $0.327^{* *}$ \\
\hline \multirow[t]{2}{*}{ PG } & $\mathbf{P}$ & 0.141 & -0.137 & 0.000 & 0.000 & -0.004 & 0.000 & 0.015 & -0.015 & 0.000 & 0.009 & -0.009 & 0.282 & 0.001 & -0.001 & 0.036 & 0.000 & 0.000 & 0.000 & $0.317 * *$ \\
\hline & G & 0.072 & -0.102 & 0.000 & 0.000 & 0.036 & 0.000 & -0.059 & 0.062 & -0.002 & 0.186 & -0.187 & 0.391 & 0.010 & -0.010 & 0.035 & -0.005 & 0.000 & 0.000 & $0.428 * *$ \\
\hline \multirow[t]{2}{*}{ PH } & $\mathbf{P}$ & -0.003 & 0.004 & 0.001 & 0.000 & 0.000 & -0.001 & 0.024 & -0.024 & 0.000 & 0.007 & -0.007 & 0.156 & 0.000 & 0.000 & 0.020 & 0.000 & 0.000 & 0.000 & 0.176 \\
\hline & G & -0.003 & 0.004 & -0.003 & -0.001 & -0.002 & 0.002 & -0.118 & 0.123 & -0.002 & 0.113 & -0.113 & 0.161 & 0.007 & -0.008 & 0.031 & 0.001 & 0.000 & -0.001 & $0.192 *$ \\
\hline \multirow[t]{2}{*}{ LMR } & $\mathbf{P}$ & 0.035 & -0.034 & 0.000 & 0.000 & -0.001 & 0.000 & 0.060 & -0.060 & 0.000 & 0.019 & -0.020 & 0.570 & 0.002 & -0.002 & 0.136 & 0.000 & 0.000 & 0.000 & $0.705^{* *}$ \\
\hline & G & 0.018 & -0.026 & -0.001 & -0.001 & 0.009 & 0.001 & -0.240 & 0.252 & -0.006 & 0.339 & -0.339 & 0.673 & 0.034 & -0.036 & 0.163 & -0.001 & 0.000 & 0.000 & $0.838 * *$ \\
\hline \multirow[t]{2}{*}{ CMR } & $\mathbf{P}$ & 0.035 & -0.033 & 0.000 & 0.000 & -0.001 & 0.000 & 0.060 & -0.060 & 0.000 & 0.019 & -0.020 & 0.570 & 0.002 & -0.002 & 0.135 & 0.000 & 0.000 & 0.000 & $0.704 * *$ \\
\hline & G & 0.019 & -0.026 & -0.001 & -0.001 & 0.009 & 0.001 & -0.241 & 0.251 & -0.006 & 0.340 & -0.339 & 0.674 & 0.034 & -0.036 & 0.163 & -0.001 & 0.000 & 0.000 & $0.839 * *$ \\
\hline \multirow[t]{2}{*}{ WMR } & $\mathbf{P}$ & 0.034 & -0.033 & 0.000 & 0.000 & -0.001 & 0.000 & 0.057 & -0.057 & 0.000 & 0.018 & -0.019 & 0.578 & 0.002 & -0.002 & 0.118 & 0.000 & 0.000 & 0.000 & $0.696^{* *}$ \\
\hline & G & 0.020 & -0.029 & -0.001 & 0.000 & 0.010 & 0.001 & -0.231 & 0.241 & -0.006 & 0.334 & -0.334 & 0.690 & 0.028 & -0.030 & 0.140 & -0.002 & 0.000 & 0.000 & $0.832 * *$ \\
\hline \multirow[t]{2}{*}{ LPR } & $\mathbf{P}$ & 0.043 & -0.042 & 0.000 & 0.000 & -0.001 & 0.000 & 0.040 & -0.040 & 0.000 & 0.029 & -0.030 & 0.895 & 0.001 & -0.001 & 0.081 & 0.000 & 0.000 & 0.000 & $0.975 * *$ \\
\hline & G & 0.031 & -0.044 & -0.001 & 0.000 & 0.015 & 0.000 & -0.185 & 0.194 & -0.005 & 0.440 & -0.440 & 0.903 & 0.017 & -0.018 & 0.078 & -0.002 & 0.000 & 0.000 & $0.983 * *$ \\
\hline \multirow[t]{2}{*}{ CPR } & $\mathbf{P}$ & 0.042 & -0.040 & 0.000 & 0.000 & -0.001 & 0.000 & 0.040 & -0.040 & 0.000 & 0.028 & $-\mathbf{0 . 0 3 0}$ & 0.894 & 0.001 & -0.001 & 0.081 & 0.000 & 0.000 & 0.000 & $0.974 * *$ \\
\hline & G & 0.031 & -0.044 & -0.001 & 0.000 & 0.015 & 0.000 & -0.186 & 0.194 & -0.005 & 0.440 & -0.439 & 0.903 & 0.017 & -0.018 & 0.078 & -0.002 & 0.000 & 0.000 & $0.983 * *$ \\
\hline
\end{tabular}




\begin{tabular}{|c|c|c|c|c|c|c|c|c|c|c|c|c|c|c|c|c|c|c|c|c|}
\hline Traits & & EMG & NTP & NLP & $\mathbf{L L}$ & PG & PH & LMR & CMR & WMR & LPR & CPR & WPR & LSR & CSR & WSR & IRR & DMR & $\mathrm{CC}$ & CYPP \\
\hline \multirow[t]{2}{*}{ WPR } & $\mathbf{P}$ & 0.044 & -0.042 & 0.000 & 0.000 & -0.001 & 0.000 & 0.038 & -0.038 & 0.000 & 0.028 & -0.030 & 0.903 & 0.001 & -0.001 & 0.070 & 0.000 & 0.000 & 0.000 & $0.973 * *$ \\
\hline & G & 0.032 & -0.045 & -0.001 & 0.000 & 0.015 & 0.000 & -0.178 & 0.187 & -0.005 & 0.437 & -0.437 & 0.908 & 0.015 & -0.015 & 0.065 & -0.002 & 0.000 & 0.000 & $0.975^{* *}$ \\
\hline \multirow[t]{2}{*}{ LSR } & $\mathbf{P}$ & 0.023 & -0.023 & 0.000 & 0.000 & -0.001 & 0.000 & 0.035 & -0.034 & 0.000 & 0.010 & -0.011 & 0.284 & 0.004 & -0.003 & 0.238 & 0.000 & 0.000 & 0.000 & $0.523 * *$ \\
\hline & G & 0.015 & -0.022 & 0.000 & 0.000 & 0.007 & 0.000 & -0.174 & 0.182 & -0.004 & 0.162 & -0.162 & 0.284 & 0.047 & -0.049 & 0.228 & -0.001 & 0.000 & 0.000 & $0.513^{* *}$ \\
\hline \multirow[t]{2}{*}{ CSR } & $\mathbf{P}$ & 0.025 & -0.025 & 0.000 & 0.000 & -0.001 & 0.000 & 0.035 & -0.034 & 0.000 & 0.010 & -0.010 & 0.276 & 0.004 & -0.003 & 0.237 & 0.000 & 0.000 & 0.000 & $0.513 * *$ \\
\hline & G & 0.015 & -0.021 & 0.000 & 0.000 & 0.007 & 0.000 & -0.174 & 0.183 & -0.004 & 0.159 & -0.161 & 0.280 & 0.047 & -0.049 & 0.228 & -0.001 & 0.000 & 0.000 & $0.509 * *$ \\
\hline \multirow[t]{2}{*}{ WSR } & $\mathbf{P}$ & 0.021 & -0.020 & 0.000 & 0.000 & -0.001 & 0.000 & 0.034 & -0.034 & 0.000 & 0.010 & -0.010 & 0.262 & 0.004 & -0.003 & 0.242 & 0.000 & 0.000 & 0.000 & $0.504 * *$ \\
\hline & $\mathbf{G}$ & 0.011 & -0.016 & 0.000 & 0.000 & 0.005 & 0.000 & -0.169 & 0.177 & -0.004 & 0.147 & -0.148 & 0.256 & 0.046 & -0.049 & 0.231 & -0.001 & 0.000 & 0.000 & $0.488 * *$ \\
\hline \multirow[t]{2}{*}{ IRR } & $\mathbf{P}$ & -0.135 & 0.131 & 0.000 & 0.000 & 0.003 & 0.000 & -0.016 & 0.016 & 0.000 & -0.009 & 0.009 & -0.296 & -0.001 & 0.001 & -0.035 & 0.001 & 0.000 & 0.000 & $-0.331 * *$ \\
\hline & G & -0.072 & 0.101 & 0.000 & 0.000 & -0.036 & 0.000 & 0.061 & -0.065 & 0.002 & -0.186 & 0.187 & -0.394 & -0.011 & 0.012 & -0.042 & 0.005 & 0.000 & 0.000 & $-0.437 * *$ \\
\hline \multirow[t]{2}{*}{ DMR } & $\mathbf{P}$ & -0.026 & 0.026 & 0.000 & 0.000 & 0.001 & 0.000 & -0.004 & 0.004 & 0.000 & 0.004 & -0.005 & 0.115 & -0.001 & 0.001 & -0.057 & 0.000 & 0.000 & 0.000 & 0.058 \\
\hline & G & -0.016 & 0.023 & -0.002 & -0.001 & -0.008 & 0.001 & 0.013 & -0.014 & 0.000 & 0.084 & -0.084 & 0.147 & -0.015 & 0.016 & -0.067 & 0.002 & 0.000 & 0.000 & 0.081 \\
\hline \multirow[t]{2}{*}{$\mathrm{CC}$} & $\mathbf{P}$ & 0.003 & -0.003 & 0.000 & 0.000 & 0.000 & 0.000 & -0.017 & 0.017 & 0.000 & -0.007 & 0.007 & -0.162 & -0.001 & 0.001 & -0.044 & 0.000 & 0.000 & 0.000 & $-0.205^{*}$ \\
\hline & $\mathbf{G}$ & 0.002 & -0.003 & 0.002 & 0.001 & 0.001 & -0.001 & 0.081 & -0.085 & 0.002 & -0.121 & 0.122 & -0.192 & -0.009 & 0.009 & -0.051 & 0.000 & 0.000 & 0.001 & $-0.244 * *$ \\
\hline
\end{tabular}

*Significant at $5 \%$ level of significance

**Significant at $1 \%$ level of significance

Where, $\mathrm{EMG}=$ Emergence, $\mathrm{NTP}=$ Number of tillers per plant, NLP= Number of leaves per plant, LL= Leaf length, $\mathrm{PG}=\mathrm{Plant}$ girth, $\mathrm{PH}=\mathrm{Plant}$ height, $\mathrm{LMR}=$ Length of mother rhizome, $\mathrm{CMR}=$ Core diameter of mother rhizome, WMR=Weight of mother rhizome, $\mathrm{LPR}=\mathrm{Length}$ of primary rhizome, $\mathrm{CPR}=\mathrm{Core}$ diameter of primary rhizome, WPR $=$ Weight of primary rhizome, LSR= Length of secondary rhizome, CSR= Core diameter of secondary rhizome, WSR $=$ Weight of secondary rhizome, IRR= Incidence of rhizome rot, $\mathrm{DMR}=$ Dry matter recovery, $\mathrm{CC}=\mathrm{Curcumin}$ content and $\mathrm{CYPP}=\mathrm{Correlation}$ coefficient for yield per plant Residual effect of phenotypic path and genotypic path: 0.000

Diagonal figures represent the direct effect 
The phenotypic and genotypic correlation coefficients were partitioned into direct and indirect effects via various yield contributing characters (Table 2). The path coefficient analysis at phenotypic level revealed that weight of primary rhizome has maximum positive direct effect on yield per plant followed by weight of secondary rhizome, emergence, length of mother, primary and secondary rhizomes and number of leaves per plant. While, negative direct effect of number of tillers per plant, plant girth, core diameter of mother, primary and secondary rhizomes was observed on yield per plant.

The data pertaining to genotypic path revealed that weight of primary rhizome has maximum positive direct effect on yield per plant followed by length of primary rhizome, core diameter of mother rhizome, weight and length of secondary rhizome, emergence, plant girth and height. While, negative direct effect of core diameter of primary rhizome, length of mother rhizome, number of tillers and leaves per plant, core diameter of secondary rhizome and leaf length was observed on yield per plant. These findings showed that selection should be made on the basis of weight of primary rhizome, having the highest positive direct effect taking other traits into consideration, while making improvement in yield of turmeric. Earlier workers like Singh and Tiwari (1995), Hazra et al., (2002), Chattopadhyay et al., (2004), Yadav et al., (2006) and Sharon et al., (2011) have also reported similar effects of component traits on yield.

\section{References}

Al-Jibouri HW, Miller PA and Robinson HF. 1958. Genotypic and environmental variances and co-variances in an upland cotton cross of interspecific origin. Agron Journal 50: 633-36.

Anonymous. 2009. Package of Practices for Vegetable Crops. Directorate of
Extension Education, Dr. YS Parmar University of Horticulture and Forestry, Nauni, Solan. 202p.

Anonymous. 2017-2018. Indian Horticulture Database http://www.nhb.gov.in [5:00 PM, $27^{\text {th }}$ May 2019].

Chandra R, Sheo G and Desai AR. 1999. Growth, yield and quality performance of turmeric (Curcuma longa L.) genotypes in mid altitudes of Meghalaya. Journal of Applied Horticulture Lucknow 1: 142-44.

Chattopadhyay N, Hore JK and Bandyopadhyay A. 2004. A Studies on character association and genetic variability in turmeric. Horticultural Journal 17: 25966.

Chaudhary AS, Sachan SK and Singh RL. 2006. Studies on varietal performance of turmeric (Curcuma longa L.) Indian Journal Crop Science 1: 189-90.

Dewey JR and Lu KH. 1959. Correlation and path analysis of components of crested wheat grass seed production. Agron Journal 51: 515-18.

Hazra P, Roy A and Bandyopadhyay A. 2002. Growth characters as rhizome yield components of turmeric (Curcuma longa L.). Crop Research Hisar 19: 235-40.

Jalgaonkar R, Jamdagni BM and Salvi MJ. 1990. Genetic variability and correlation studies in turmeric. Indian Cocoa, Arecanut and Spices Journal 14: 20-22.

Jana JC, Dutta S and Chatterjee R. 2001. Genetic variability, heritability and correlation studies in turmeric (Curcuma longa L.). Research on Crops 2: 220-25.

Kumar RA, Vijayalatha KR, Alagesan A and Chezhiyan N. 2006. Correlation and path coefficient analysis for economic traits in turmeric (Curcuma longa) genotypes. Journal of Ecobiology 19: 307-12.

Kumar RA, Vijayalatha KR, Alagesan A and Chezhiyan N. 2007. Correlation and path coefficient for morphological traits and yield in turmeric (Curcuma longa) genotypes. Journal of Ecobiology 19: 3336.

Lynrah PG, Barua PK and Chakrabarty BK. 1998. Pattern of genetic variability in a 
collection of turmeric (Curcuma spp.) genotypes. Indian Journal of Genetics and Plant Breeding 58: 201-07.

Maurya KR. 1990. RH-10-A promising variety of turmeric to boost farmer's economy. Indian Cocoa, Arecanut and Spices Journal 13:100-01.

Nandi A, Lenka D and Singh DN. 1994. Path analysis in turmeric (Curcuma longa $\mathrm{L}$ ). Indian Cocoa, Arecanut and Spices Journal 18: 54-55.

Panja B, De DK, Basak S and Chattopadhyay SB. 2002. Correlation and path analysis in turmeric (Curcuma longa L.). Journal of Spices and Aromatic Crops 11: 70-73.

Pathania NK, Arya PS and Korla BN. 1981. Path analysis in turmeric (Curcuma longa Linn.). Madras Agricultural Journal 68: 675-78.

Prasad R, Yadav LM and Yadav RC. 2004. Correlation study in turmeric. Journal of Applied Biology 14: 44-45.

Rao AM, Rao PV, Reddy YN and Ganesh M. 2004. Variability and correlation studies in turmeric (Curcuma longa L.). Crop Research Hisar 27: 275-81.

Raveendra BH, Hanamashetti SI and Hegde LN. 2001. Correlation studies with respect to growth and yield of sixteen cultivars of turmeric (Curcuma longa L.). Journal of Plantation Crops 29: 61-63.

Sharon A, Shoba N, Rajamani K and Manonmani S. 2011. Correlation studies in turmeric (Curcuma longa L.). Research on Crops 12: 195-97.

Shashidhar TR and Sulikeri GS. 1997. Correlation studies in turmeric (Curcuma longa L.). Karnataka Journal of Agricultural Sciences 10: 595-97.
Shashidhar TR, Sulkeri GS and Gasti VD. 1997. Correlation studies in turmeric (Curcuma longa L.). Mysore Journal of Agricultural Sciences 31: 217-20.

Singh AP, Pandey VP, Rahman SMA and Pervez R. 2012. Genetic variability and character association in turmeric (Curcuma longa L.). Trends in Biosciences 5: 11-13.

Singh DP and Tiwari RS. 1995. Path analysis in turmeric (Curcuma longa L.). Recent Horticulture 2: 113-16.

Stahl WH. 1980. Turmeric-Chemistry, Technology and Quality In: CRC Critical reviews in Food, Science and Nutrition. pp. 199-200.

Tomar NS, Nair SK and Gupta CR. 2005. Character association and path analysis for yield components in turmeric (Curcuma longa L.). Journal of Spices and Aromatic Crops 14: 75-77.

Velmurugan $\mathrm{M}$, Chezhiyan $\mathrm{N}$ and Jawaharlal M. 2008. Correlation and path analysis in turmeric cv. BSR-2. International Journal of Agricultural Sciences 4: 103-06.

Venkatesha J, Khan MM and Chandrappa H. 1998. Studies on character association in turmeric. Developments in plantation crops research Proceedings of the $12^{\text {th }}$ Symposium on Plantation Crops, PLACROSYM-XII, Kottayam, India, 2729 November 1996: 54-57.

Yadav RK, Yadav DS, Rai N, Asati BS and Singh AK. 2006. Correlation and path coefficient analysis in turmeric (Curcuma longa L.). Indian Journal of Horticulture 63: 103-06.

\section{How to cite this article:}

Dev, H. and Vipin Sharma. 2020. Correlation Studies in Turmeric (Curcuma longa L.). Int.J.Curr.Microbiol.App.Sci. 9(08): 3063-3071. doi: https://doi.org/10.20546/ijcmas.2020.908.346 